Artículo

\title{
Nanoparticulas de óxido de zinc y su efecto en el rendimiento y calidad de melón
}

\author{
Rubén Guadalupe Rivera-Gutiérrez ${ }^{1}$ \\ Pablo Preciado-Rangel ${ }^{1}$ \\ Manuel Fortis-Hernández ${ }^{1 \S}$ \\ Rebeca, Betancourt-Galindo ${ }^{2}$ \\ Pablo Yescas-Coronado ${ }^{1}$ \\ Jorge Arnaldo Orozco-Vidal ${ }^{1}$
}

${ }^{1}$ Tecnológico Nacional de México-Campus Instituto Tecnológico de Torreón. Antigua carretera TorreónSan Pedro km 7.5, Torreón, Coahuila, México. CP. 27170. Tel. 871 7507199. (ing.ruben_faz@ hotmail.com; ppreciador@yahoo.com.mx; joorvi66@hotmail.com; pyescas@ hotmail.com). ${ }^{2}$ Centro de Investigación en Química Aplicada. Enrique Reyna H. núm. 140, San José de los Cerritos, Saltillo, Coahuila, México. CP. 25294. Tel. 844 4389830. (rebeca.betancourt@ ciqa.edu.mx).

${ }^{\S}$ Autor para correspondencia: fortismanuel@ hotmail.com.

\section{Resumen}

La investigación consistió en la aplicación foliar de nanopartículas de óxido de zinc (NPs ZnO), dos fuentes comerciales (Z40 y GZ), además de un tratamiento testigo y su efecto en el rendimiento y calidad comercial y nutracéutica en frutos de melón (Cucumis melo L.) cv Cruiser. Los tratamientos consistieron en la aplicación foliar de cinco dosis de NPs ZnO: 50, 100, 150, 200 y $250 \mathrm{mg} \mathrm{L}^{-1}$. Los resultados indicaron que el mayor rendimiento fue para el tratamiento con $200 \mathrm{mg} \mathrm{L}^{-1}$ de NPs ZnO con un incremento de $53 \mathrm{t} \mathrm{ha}^{-1}$, con relación al testigo, misma tendencia fue para el peso del fruto que mostró valores de $1.74 \mathrm{~kg}, 1.45 \mathrm{~kg}$ y $1.33 \mathrm{~kg}$, respectivamente. En relación con el contenido de flavonoides, las dosis de 50 y $100 \mathrm{mg} \mathrm{L}^{-1}$ superaron al tratamiento testigo y a las fuentes comerciales; sin embargo, a medida que aumentaba la concentración de $\mathrm{NP}$, disminuían los flavonoides. Los fenoles, la capacidad antioxidante (DPPH) y la vitamina C disminuyeron en 17, 10.8 y 9\% con el control respectivamente. La mayor concentración de zinc en pulpa fue observada con e $200 \mathrm{mg} \mathrm{L}^{-1} \mathrm{NPs} \mathrm{ZnO}$. El uso de nanopartículas de zinc es una buena alternativa para mejorar el rendimiento y contenido nutracéutico y la concentración de $\mathrm{Zn}$ en frutos de melón, siendo una opción para combatir la desnutrición de este micronutrimento.

Palabras clave: Cucumis melo L., DPPH, fenoles, flavonoides, nanopartículas.

Recibido: mayo de 2021

Aceptado: agosto de 2021 


\section{Introducción}

El melón (Cucumis melo L.) es una de las hortalizas que se cultiva en muchas regiones del mundo, en el año 2019 se reportaron 1039691 hectáreas, siendo China, Irán y Turquía, los principales países productores (FAOSTAT, 2020). En México se siembra en lugares que presentan climas cálidos y escasa precipitación, en el año 2019 se reportaron cerca de 20000 ha (SIAP, 2021). En el norte del país se localiza el estado de Coahuila, donde 1800 productores sembraron más de 6 mil ha con un alto grado de especialización, calidad e inocuidad, lo que ha permitido su exportación (Arellano et al., 2017).

El melón es un fruto caracterizado por aportar una cantidad importante de beneficios tanto para la salud como para la economía, razón por la cual es uno de los más consumidos a nivel mundial. La pulpa de melón es rica en agua, proteínas, lípidos, fuente de vitamina $\mathrm{C}$ y beta-carotenos (Maietti, 2012). Es una fuente de antioxidantes y polifenoles bioactivos, que proporcionan beneficios importantes para la salud, particularmente para el sistema cardiovascular (López et al., 2007). Además, es ligeramente diurético, ya que elimina las toxinas del cuerpo (Bayer, 2018).

El uso y aplicación de nanopartículas metálicas en la agricultura ha sido exitosa, sobre todo en cultivos que se producen en climas secos, ya que estimulan su crecimiento, incrementan el rendimiento y la calidad comercial de sus frutos (Prasad et al., 2014). También se reporta que inducen el estrés oxidativo en las plantas (Chandra et al., 2020) y tienen influencia en la actividad fotosintética y antioxidante (Kumar et al., 2020). En general, los efectos de los nanomateriales en las plantas varían mucho no solo por la naturaleza de los materiales sino dependiendo de la especie estudiada (Wang et al., 2015). El zinc, es un micronutriente esencial para las plantas y los seres humanos, a nivel mundial más de $30 \%$ de la población tiene deficiencia de este elemento (Amarakoon et al., 2012), afectando negativamente el desarrollo cognitivo, cerebral y reproductivo de las personas (Salgueiro et al., 2004).

Tiene un efecto directo en el crecimiento, desarrollo neurológico, conductual y en el sistema inmunológico (López de Romaña et al., 2010) además, promueve el desarrollo intelectual de los niños, acelera el crecimiento de los adolescentes, afecta el paladar, el apetito y la fertilidad masculina (Yin et al., 2012). Se han propuesto estrategias para atenuar este problema, una de ellas ha sido la biofortificación donde es posible obtener frutos enriquecidos con minerales (Restrepo et al., 2020). Reportes han mostrado que es posible obtener frutos biofortificados con minerales, incrementando su concentración en las porciones comestibles de las plantas (Neeraja et al., 2017).

Para el caso del zinc en las plantas, este es un micronutriente involucrado en la síntesis de proteínas, es un agente estabilizador para las membranas de amplia propagación y alargamiento celular (Mousavi et al., 2014), mantienen una protección contra el estrés ambiental (Sturikova et al., 2018), y ayuda a la adaptabilidad a las duras condiciones ambientales (Cakmak, 2017). La aplicación de las Nanopartículas de óxido de zinc (NPs ZnO), han demostrado tener efectos positivos sobre parámetros nutricionales y fisiológicos en algunos cultivos (Rizwan et al., 2018), además de incrementar su contenido en la parte comestible de la planta (Subbaiah et al., 2016), estos cambios pueden inducir estrés y toxicidad en las plantas y estimular los sistemas antioxidantes (GarcíaGómez et al., 2017). 
Las NPs ZnO aplicadas vía foliar minimizan el uso de fertilizantes, aumentan la eficiencia y mayor disponibilidad de nutrientes en las plantas (Nandhini et al., 2019), además son mayores los efectos sobre el crecimiento y desarrollo de las plantas en comparación con las formas tradicionales de $\mathrm{Zn}$ (Kolěncík et al., 2019). Sin embargo, la información de los efectos de su aplicación de esta manera aún es escasa, por lo que el objetivo de esta investigación fue determinar el efecto de la aspersión foliar de nanopartículas de zinc en la producción, calidad nutracéutica y su concentración de este micronutrimento en frutos de melones desarrollados en condiciones de campo abierto.

\section{Materiales y métodos}

\section{Sitio de estudio}

El experimento se realizó en suelo agrícola en el ejido Rosas, Municipio de Tlahualilo, Durango, México, se encuentra a $1095 \mathrm{msnm}$. Se localiza en las coordenadas $26^{\circ} 06^{\prime} 38^{\prime}$ ' latitud norte y $103^{\circ} 26^{\prime} 17^{\prime}$ ' longitud oeste. Según la clasificación de Köppen modificado por García (2004): clima seco desértico o estepario cálido con lluvias en el verano e inviernos frescos. La precipitación pluvial es de $258 \mathrm{~mm}$ y la temperatura media anual es de $22.1^{\circ} \mathrm{C}$, con rangos de 38.5 como media máxima y 16.1 como media mínima. La evaporación anual media es de $2396 \mathrm{~mm}$.

\section{Nanopartículas de óxido de zinc}

Las nanopartículas utilizadas fueron de óxido de zinc (NPs ZnO), con un tamaño entre 20 y $60 \mathrm{~nm}$, una pureza de $97 \%$, de color blanco y de forma estructural semiesférica y poligonal (Figura 1). Las NPs ZnO fueron sintetizadas a través de precipitación controlada de acuerdo con lo reportado por (Ramírez Barrón et al., 2019), mediante el método de hidrólisis química. $530.4 \mathrm{~g}$ de acetato de zinc $\left(\mathrm{CH}_{3} \mathrm{CO}_{2}\right)_{2} \mathrm{Zn}$ y $24 \mathrm{~L}$ de etanol se virtieron en un reactor de $200 \mathrm{~L}$ con calentamiento mediante una caldera a una temperatura de $80{ }^{\circ} \mathrm{C}$, utilizando un controlador de temperatura. La reacción se mantiene en agitación durante $3 \mathrm{~h}$.

Posteriormente se adiciona al reactor una solución de $0.22 \mathrm{M}$ de $\mathrm{NaOH}$. Se deja en agitación durante $1 \mathrm{~h}$ para lograr la precipitación de las NPs. El precipitado obtenido se lavó varias veces con agua destilada para su posterior caracterización mediante microscopía electrónica de transmisión (TEM) para determinar el tamaño de partícula del material obtenido.

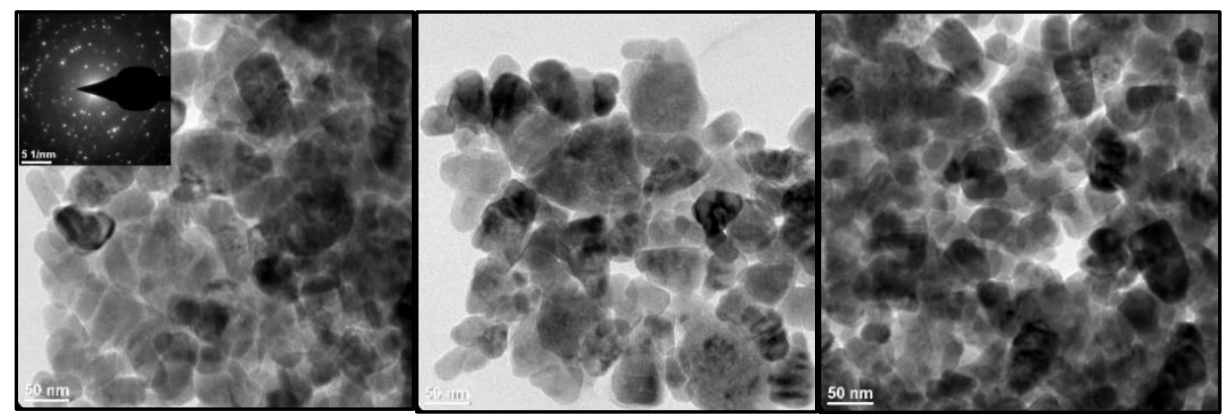

Figura 1. Forma estructural de las nanopartículas de óxido de zinc (NPs ZnO). 


\section{Material vegetal y cultivo}

El material vegetativo utilizado fue melón (Cucumis melo L.) híbrido cv crusier tipo cantaluop (Harris Moran Seed Companyr $^{\circledR}$ ). La siembra directa se realizó el 20 de marzo de 2019. Se construyeron bordos a doble hilera formando una micro-parcela (cama melonera) a una distancia de $4 \mathrm{~m}$ entre bordos y una separación entre plantas de $30 \mathrm{~cm}$ para una densidad de 16665 plantas por hectárea. De acuerdo con el paquete tecnológico para este cultivo (Cano et al., 1992), se realizó la preparación de suelo que consistió en barbecho a $30 \mathrm{~cm}$ de profundidad, doble rastreo, seguido de una nivelación, construcción de camas meloneras y bordos para conducción y retención del agua de riego.

La fertilización fue mecánica utilizando la fórmula química 120-60-00 ( $\left.\mathrm{N}_{-} \mathrm{P}_{2} \mathrm{O}_{5}-\mathrm{K}_{2} \mathrm{O}\right)$; se incorporó todo el fósforo y la mitad del nitrógeno al momento de la siembra y el resto del nitrógeno al momento de la floración. Los fertilizantes fueron fosfato monoamónico $\left(\mathrm{NH}_{4} \mathrm{H}_{2} \mathrm{PO}_{4}\right)$ y sulfato de amonio $\left[\left(\mathrm{NH}_{4}\right)_{2} \mathrm{SO}_{4}\right]$. El riego fue con agua de la presa Francisco Zarco, se aplicó un riego de presiembra de $30 \mathrm{~cm}$, durante el ciclo de cultivo se dieron seis riegos de auxilio con láminas de 15 cm cada uno, en total se aplicó una lámina de $120 \mathrm{~cm}$.

\section{Tratamientos y diseño experimental}

El diseño experimental fue bloques al azar considerando ocho tratamientos con cuatro repeticiones, conformando 32 unidades experimentales, cada una de ellas constituida por un área de $4 \mathrm{~m}$ de ancho por $10 \mathrm{~m}$ de largo $\left(40 \mathrm{~m}^{2}\right)$. Las concentraciones de NPs $\mathrm{ZnO}$ aplicadas fueron las siguientes: $\mathrm{T} 1=$ control, $\mathrm{T} 2=50 \mathrm{mg} \mathrm{L}^{-1} ; \mathrm{T} 3=100 \mathrm{mg} \mathrm{L}^{-1} ; \mathrm{T} 4=150 \mathrm{mg} \mathrm{L}^{-1} ; \mathrm{T} 5=200 \mathrm{mg} \mathrm{L}^{-1} ; \mathrm{T} 6=250 \mathrm{mg} \mathrm{L}^{-1}$; $\mathrm{T} 7=\mathrm{Z} 40\left(1 \mathrm{~L} \mathrm{ha}^{-1}\right)$ y $\mathrm{T} 8=\mathrm{GZ}\left(1 \mathrm{ml} \mathrm{L}^{-1}\right.$ de agua $)$. Los tratamientos $\mathrm{T} 7 \mathrm{y} \mathrm{T}$, consistieron en la aplicación de fertilizantes foliares comerciales, el zinc 40 (Z40: concentración 40\%) Tec-Fort ${ }^{\circledR}$ y Gro-zinc (GZ: concentración 9.66\%) Grodeco ${ }^{\circledR}$, ambos fertilizantes quelatados.

Las nanopartículas fueron aplicadas con una aspersora manual Lola de 20 L (Swissmex ${ }^{\circledR}$ ). Cada concentración de NPs ZnO se disolvió en ocho litros de agua desionizada (Quimicrón ${ }^{\circledR}$ ) y para una mejor adherencia se le agrego un acidificante - adherente no iónico (AF-optimus ${ }^{\circledR}$ ) a una dosis de $1-2 \mathrm{ml} \mathrm{L}{ }^{-1}$ de agua de aspersión. Se realizaron tres aplicaciones foliares de NPs $\mathrm{ZnO}$ y de los fertilizantes convencionales, la primera a los 20 días después de la siembra, cuando el cultivo presentaba expansión del follaje, posteriormente cada 15 días, en promedio fueron aplicados $40 \mathrm{ml}$ por planta. Las aplicaciones fueron realizadas por la mañana, sin presencia de viento y mojaron toda la planta y (Trinidad y Aguilar, 1999).

\section{Variables evaluadas}

\section{Peso, solidos solubles y firmeza de fruto}

Para el análisis de todas las variables se consideraron ocho plantas por tratamiento, dos por cada repetición y los frutos cosechados considerados fueron aquellos que presentaban madurez comercial; es decir, estado firme-maduro o 3/4 desprendido, entre 1.2-1.5 kg y sin daños físicos (Beaulieu yJeanne, 2007). Para el peso de fruto (PF) se pesaron todos los frutos cosechados en una báscula digital (Adir ${ }^{\circledR}$, USA) con capacidad de 5000 g. Los sólidos solubles totales (SST) se midieron con un Refractómetro óptico Master T 0-53 brix AT-Master53T (Twiligth ${ }^{\circledR}$, USA), para 
ello se partieron los frutos y se colocó una gota de jugo en el vidrio y se tomó la lectura. Los resultados se expresaron como ${ }^{\circ}$ Brix. La firmeza del fruto (FF) se midió en dos melones de cada repetición con un Penetrómetro marca Extech ${ }^{\circledR}$ Instruments modelo FHT200 (USA), se utilizó la punta o embolo de $8 \mathrm{~mm}$. Se quitó el exocarpio (cáscara) y éstos fueron colocados sobre una superficie plana, se realizaron cuatro penetraciones por fruto (Azam et al., 2015) y se promediaron los resultados registrándose como fuerza máxima de compresión $(\mathrm{N})$.

\section{Rendimiento}

Para esta variable se consideró el peso de todos los frutos cosechados por unidad experimental, la densidad de siembra y se extrapolo para una hectárea, los resultados fueron reportados en $\mathrm{t} \mathrm{ha}^{-1}$.

\section{Flavonoides totales (FLVT)}

El contenido total de flavonoides se determinó mediante el método colorimétrico (Zhishen et al., 1999) y los resultados se expresaron en mg equivalentes de quercetina por $100 \mathrm{~g}$, basado en el peso fresco (mg equiv Q $100 \mathrm{~g}^{-1} \mathrm{PF}$ ).

\section{Compuestos fenólicos totales}

El contenido fenólico total se midió mediante el método de Folin-Ciocalteau (Singleton et al., 1999). Las determinaciones se realizaron por triplicado y fueron reportadas en $\mathrm{mg}$ de ácido gálico equivalente por $100 \mathrm{~g}$ de peso fresco (mg equiv GA $100 \mathrm{~g}^{-1} \mathrm{PF}$ ).

\section{Actividad antioxidante}

La capacidad antioxidante se determinó utilizando el método de DPPH (1,1-difenil-2-picrilo hidracilo), desarrollado por Brand-Willams et al. (1995). Los análisis se hicieron por triplicado y se reportaron en $\mu \mathrm{M}$ equivalente de Trolox por $100 \mathrm{~g}$ de peso fresco ( $\mu \mathrm{M}$ equiv Trolox $100 \mathrm{~g}^{-1} \mathrm{PF}$ ).

\section{Vitamina C}

El ácido ascórbico se determinó por la técnica de Doner y Hicks (1981). Las lecturas se realizaron en un HPLC Varian ProStar 320 equipado con un detector UV-Vis (ProStar 210, Varian Prostar Inc., Walnut griega, CA, EE. UU.), utilizando una columna Varian amina de $10 \mathrm{~cm}$ y un bucle de inyección de $20 \mathrm{ml}$, las unidades se reportan en $\mathrm{mg}$ por 100 gramos de peso fresco $\left(\mathrm{mg} 100 \mathrm{~g} \mathrm{~g}^{-1} \mathrm{PF}\right)$.

\section{Contenido de zinc en fruto}

Se determinó el contenido de zinc en la cascara, pulpa y semillas de seis frutos de melón por tratamiento. El método utilizado fue por espectrofotometría de absorción atómica (AOAC, 1995), utilizando un equipo marca Perkin Elmer ${ }^{\circledR}$ modelo 3110. Los resultados se reportaron en $\mathrm{mg} \mathrm{kg}^{-1}$.

\section{Análisis estadístico}

Los datos de las variables fueron analizados mediante análisis de varianza y prueba de separación de medias utilizando la prueba de Tukey $(p \leq 0.05)$ con el paquete estadístico Statistical Analysis System Institute (SAS) versión 9.1. 


\section{Resultados y discusión}

\section{Parámetros de calidad de frutos de melón}

Las aplicaciones foliares de NPs de $\mathrm{ZnO}$ tuvieron efectos favorables en los frutos de melón, la concentración de $200 \mathrm{mg} \mathrm{L}^{-1}$ la que promovió mayor peso de frutos, rendimiento, solidos solubles y firmeza, superando en $32 \%$ al tratamiento control y con respecto a las fuentes comerciales de $\mathrm{Zn}$ (Z-40 y GZ) los superó $24 \%$ y $20 \%$, respectivamente. Por otra parte, la concentración de $250 \mathrm{mg}$ $\mathrm{L}^{-1}$ disminuyó $11 \%$ el peso del fruto (Cuadro 1). Respecto a rendimiento el tratamiento de $200 \mathrm{mg}$ $\mathrm{L}^{-1}$, reporto el mayor rendimiento con $53 \mathrm{t} \mathrm{ha}^{-1}$, el tratamiento con la fuente comercial de zinc (GZ) $43.95 \mathrm{t} \mathrm{ha}^{-1} \mathrm{y}$ el control $40 \mathrm{tha}^{-1}$.

Cuadro 1. Comparación de parámetros de calidad comercial de frutos de melón cantaloupe por efecto de la aplicación foliar de NPs ZnO en diferentes concentraciones.

\begin{tabular}{ccccc}
\hline $\begin{array}{c}\text { NPs ZnO } \\
\left(\mathrm{mg} \mathrm{L}^{-1}\right)\end{array}$ & Peso fruto $(\mathrm{kg})$ & Rendimiento $\left(\mathrm{t} \mathrm{ha}^{-1}\right)$ & Solidos solubles $\left({ }^{\circ}\right.$ Brix $)$ & Firmeza (Newton) \\
\hline Control & $1.33 \pm 0.27 \mathrm{~d}^{*}$ & $40.09 \pm 8.35 \mathrm{~d}$ & $11.26 \pm 0.38 \mathrm{~b}$ & $17.75 \pm 1.25 \mathrm{~b}$ \\
50 & $1.49 \pm 0.11 \mathrm{bdac}$ & $44.95 \pm 3.64 \mathrm{bdac}$ & $11.37 \pm 0.22 \mathrm{~b}$ & $21.25 \pm 3.59 \mathrm{ba}$ \\
100 & $1.7 \pm 0.09 \mathrm{ba}$ & $51.63 \pm 2.93 \mathrm{ba}$ & $11.85 \pm 0.75 \mathrm{~b}$ & $22.25 \pm 3.59 \mathrm{ba}$ \\
150 & $1.61 \pm 0.23 \mathrm{bac}$ & $48.93 \pm 7.13 \mathrm{bac}$ & $11.76 \pm 1.05 \mathrm{~b}$ & $23 \pm 5.83 \mathrm{a}$ \\
200 & $1.74 \pm 0.18 \mathrm{a}$ & $52.96 \pm 5.59 \mathrm{a}$ & $12.73 \pm 0.31 \mathrm{a}$ & $24.5 \pm 6.45 \mathrm{a}$ \\
250 & $1.64 \pm 0.16 \mathrm{bac}$ & $49.73 \pm 5.03 \mathrm{bac}$ & $11.94 \pm 0.48 \mathrm{~b}$ & $20.5 \pm 5.68 \mathrm{ba}$ \\
$\mathrm{Z} 40$ & $1.4 \pm 0.17 \mathrm{dc}$ & $42.53 \pm 5.36 \mathrm{dc}$ & $11.65 \pm 0.21 \mathrm{~b}$ & $21.75 \pm 2.21 \mathrm{ba}$ \\
$\mathrm{GZ}$ & $1.45 \pm 0.17 \mathrm{bdc}$ & $43.95 \pm 5.39 \mathrm{bdc}$ & $11.6 \pm 0.13 \mathrm{~b}$ & $21.75 \pm 3.77 \mathrm{ba}$ \\
\hline
\end{tabular}

* = promedios con letras distintas, dentro de una misma columna, indican diferencia significativa (Tukey; $p \leq 0.05$ ). Donde: $\mathrm{LSD}=$ diferencia mínima significativa.

Los rendimientos medios regionales reportados para este cultivo son de $45.2 \mathrm{t} \mathrm{ha}^{-1} \mathrm{y}$ con un peso de fruto de $1.7 \mathrm{~kg}$ (García-Mendoza et al., 2019), en ambos casos las aplicaciones de las nanopartículas superaron estos valores. Resultados reportan para Vigna mungo (L.), con dosis de 200 ppm de NPs ZnO los mejores resultados para germinación de semillas y crecimiento de plantas (Rajitha et al., 2020). En lechuga, reportan una mejor respuesta al aplicar 20 ppm (NPs Ag) y al aplicar el doble disminuyo el peso fresco de las hojas (Jurkow et al., 2020).

En plantas de Capsicum chinense aplicaciones foliares de $1000 \mathrm{mg} \mathrm{L}^{-1}$ presentaron efectos positivos en el crecimiento y con $2000 \mathrm{mg} \mathrm{L}^{-1}$ existe un efecto negativo. Diversos estudios señalan que los efectos positivos de las NPs $\mathrm{ZnO}$ puede ser atribuido a que el zinc es indispensable para desarrollar actividades metabólicas y catalíticas en el crecimiento de las plantas (Fageria et al., 2016), regula actividades enzimáticas y reacciones bioquímicas que conducen a la formación de clorofila y carbohidratos (Gawrońska et al., 2018). Sin embargo, el efecto de las nanopartículas varía en función de sus propiedades como su tamaño, las sustancias químicas, su estructura, el recubrimiento superficial, velocidad y dosis de aplicación (Khodakovskaya et al., 2012).

Con respecto a las fuentes convencionales de zinc, se señala que los nanofertilizantes foliares pueden ser más efectivos que éstos debido a que su liberación puede ser lenta y gradual (DeRosa et al., 2010). Respecto a la disminución del peso y rendimiento de los frutos de melón, se ha 
señalado un efecto ambivalente de las nanopartículas (Méndez-Arguello et al., 2016). Por ejemplo, en Lycopersicon esculentum disminuyeron parámetros de crecimiento cuando se aplicaron foliarmente $200 \mathrm{mg} \mathrm{L}^{-1}$ de NPs ZnO (Faisan y Hayat, 2019). Las causas son diversas desde una posible toxicidad (Reed et al., 2011; Jurkow et al., 2020) o debido a factores vinculados a la forma, tamaño, periodo estacional y número de aplicaciones de las nanopartículas (Kolenčík et al., 2019).

Respecto a solidos solubles y firmeza, los valores reportados para esta hortaliza en la región son entre 6.5 a $11^{\circ}$ Brix y firmeza de $38 \mathrm{~N}$ (Moreno-Reséndez et al., 2014). El tratamiento de $200 \mathrm{mg} \mathrm{L}^{-1}$, supero al valor regional, pero fue inferior a firmeza. De acuerdo con estándares internacionales melones con contenidos de azúcar 12-14 ${ }^{\circ}$ Brix son los adecuados para el mercado internacional y valores de $9{ }^{\circ}$ Brix son los óptimos para la calidad interna óptima de los melones (Bower et al., 2002).

\section{Compuestos bioactivos en frutos}

Los resultados indican que el mayor contenido de flavonoides se obtuvo con las concentraciones de 50 y $100 \mathrm{mg} \mathrm{L}^{-1}$, superando al tratamiento control en $13.5 \%$ y a las dos fuentes de Zinc comercial en 10\% (Z40) y 12\% (GZ). Cabe señalar que a medida que se incrementó la concentración de las Nanopartículas los flavonoides disminuyeron en $8 \%$. Mismo comportamiento se presentó para fenoles, capacidad antioxidante (DPPH) y vitamina C, disminuyendo en $17 \%, 10.8 \%$ y $9 \%$, respectivamente (Cuadro 2).

Cuadro 2. Compuestos bioactivos presentes en frutos de melón por la aplicación foliares de NPs ZnO.

\begin{tabular}{ccccc}
\hline $\begin{array}{c}\text { NPs ZnO } \\
\left(\mathrm{mg} \mathrm{L}^{-1}\right)\end{array}$ & $\begin{array}{c}\text { Flavonoides }(\mathrm{mg} \text { QE } \\
\left.100 \mathrm{~g}^{-1} \mathrm{PF}\right)\end{array}$ & $\begin{array}{c}\text { Fenoles }(\mathrm{mg} \text { Ac. } \\
\left.\text { Gálico } 100 \mathrm{~g}^{-1} \mathrm{PF}\right)\end{array}$ & $\begin{array}{c}\text { DPPH }(\mathrm{mg} \text { equiv } \\
\left.\text { Trolox } 100 \mathrm{~g}^{-1} \mathrm{PF}\right)\end{array}$ & $\begin{array}{c}\text { Vitamina C (mg } \\
\left.100 \mathrm{~g}^{-1} \mathrm{PF}\right)\end{array}$ \\
\hline Control & $122.65 \pm 0.22 \mathrm{e}^{*}$ & $145.07 \pm 0.36 \mathrm{~g}$ & $63.86 \pm 0.29 \mathrm{~h}$ & $50.51 \pm 0.59 \mathrm{e}$ \\
50 & $141.85 \pm 0.55 \mathrm{a}$ & $231.36 \pm 1.32 \mathrm{a}$ & $88.14 \pm 0.22 \mathrm{a}$ & $61.36 \pm 1.04 \mathrm{a}$ \\
100 & $140.18 \pm 3.53 \mathrm{a}$ & $222.43 \pm 0.97 \mathrm{~b}$ & $81.79 \pm 0.77 \mathrm{c}$ & $59.13 \pm 0.83 \mathrm{~b}$ \\
150 & $135.22 \pm 0.45 \mathrm{~b}$ & $211.8 \pm 1.1 \mathrm{c}$ & $85.29 \pm 0.19 \mathrm{~b}$ & $56.87 \pm 0.31 \mathrm{c}$ \\
200 & $133.21 \pm 0.14 \mathrm{~b}$ & $199.9 \pm 3.27 \mathrm{~d}$ & $78.62 \pm 0.22 \mathrm{~d}$ & $53.53 \pm 0.99 \mathrm{~d}$ \\
250 & $130.11 \pm 0.77 \mathrm{c}$ & $192.04 \pm 1.68 \mathrm{e}$ & $75.32 \pm 0.11 \mathrm{e}$ & $54.17 \pm 1 \mathrm{~d}$ \\
$\mathrm{Z} 40$ & $127.71 \pm 0.17 \mathrm{~d}$ & $178.44 \pm 6.54 \mathrm{f}$ & $72.02 \pm 0.11 \mathrm{f}$ & $53.81 \pm 0.5 \mathrm{~d}$ \\
$\mathrm{GZ}$ & $124.41 \pm 0.72 \mathrm{e}$ & $150.98 \pm 8.39 \mathrm{~g}$ & $68.78 \pm 0.7 \mathrm{~g}$ & $55.9 \pm 0.43 \mathrm{c}$ \\
\hline
\end{tabular}

* = promedios con letras distintas, dentro de una misma columna, indican diferencia significativa (Tukey; $p \leq 0.05$ ). Donde: PF= peso fresco; DPPH=1,1-Difenil-2-Picrilhidrazilo.

Las aplicaciones de las fuentes de zinc comercial superaron al tratamiento control para flavonoides en $4 \%$, fenoles $18 \%$, capacidad antioxidante $11.3 \%$ y vitamina $\mathrm{C}$ en $9.6 \%$; sin embargo, estos porcentajes fueron inferiores a los obtenidos por las NPs $\mathrm{ZnO}$. La cantidad de flavonoides presentes en el melón dependen del genotipo, maduración del fruto y la fecha de cosecha (Tadmor et al., 2010). Tienen diversas funciones, actúa como un excelente agente antioxidante, tiene compuestos antimicrobianos, protectores UV, protectores de insectos (Harborne y Williams, 2000). 


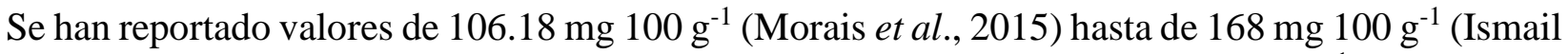
et al., 2010), en nuestro estudio los valores promedio fueron de 141.85 con $50 \mathrm{mg} \mathrm{L}^{-1}$ de NPs ZnO. Estos resultados pueden estar asociados a que la aplicación foliar de NPs ZnO tienen un mayor potencial de transporte, mayor biodisponibilidad y absorción que les permite interactuar con estructuras intracelulares que estimulan la formación de ROS (Ghosh et al., 2016; García-López et al., 2019).

Los compuestos fenólicos también desempeñan un papel muy importante en este cultivo, ya que protegen el fruto de insectos y microorganismos, y determinan el color y apariencia de los frutos (Jeong et al., 2004; Mallek-Ayadi et al., 2019). Valores de $168 \mathrm{mg} 100 \mathrm{~g}^{-1}$ se han reportado en pulpa de melón cantaloupe (Ismail et al., 2010). El contenido fenólico está asociado a sus actividades antioxidantes debido a sus propiedades redox que le permite actuar como agente reductor y donante de oxígeno (Chang et al., 2001). En este sentido, los resultados obtenidos indican claramente que la aplicación de NP de $\mathrm{ZnO}$ induce un mayor contenido de compuestos antioxidantes (fenoles, flavonoides y vitamina $\mathrm{C}$ ) en los frutos de melón.

El contenido de vitamina $\mathrm{C}$ en frutos de Melón (Cucumis melo L.) mostró un descenso al aumentar la concentración de NPs $\mathrm{ZnO}$ entre los tratamientos. El valor más alto de vitamina $\mathrm{C}$ se encontró en frutos de plantas asperjadas con $50 \mathrm{mg} \mathrm{L}^{-1}$ de NPs ZnO, esta concentración fue superior $21.48 \%$ en comparación con el tratamiento control. Las dosis de 100, 150, 200 y $250 \mathrm{mg} \mathrm{L}^{-1} \mathrm{NPs} \mathrm{ZnO}$ mostraron aumentos de 17.06, 12.59, 5.97, 7.24\%, respectivamente. Mientras que las fuentes comerciales de zinc-40 (6.53\%) y Gro-zinc (10.67\%) en comparación al tratamiento control.

Resultados obtenidos por López-Vargas et al. (2018) mostraron que aplicaciones de $250 \mathrm{mg} \mathrm{L}^{-1}$ NPs de $\mathrm{Cu}$ aumentaron significativamente la concentración de vitamina $\mathrm{C}$, lo cual aumento la calidad nutraceútica de frutos de tomate. Mathpal et al. (2015) afirman que el zinc participa en la activación de enzimas, biosíntesis de proteínas y carbohidratos. Padayatty et al. (2003) mencionan que la vitamina $\mathrm{C}$, es un antioxidante cuyos efectos aminoran las enfermedades humanas como la aterosclerosis y el cáncer mismas que ocurren por daño oxidativo a los tejidos, las dietas abundantes en frutas y verduras disminuyen el riesgo de enfermedad cardiovascular, accidente cerebrovascular y cáncer.

\section{Contenido de zinc en frutos}

Las aplicaciones foliares de NPs ZnO influyeron en el contenido de zinc presente en la cascara, pulpa y semillas de los frutos de melón. En el tratamiento control la cantidad de zinc fue de 52.68 mg kg-1 la mayor cantidad de zinc se encuentra presente en la pulpa (76\%), después en semilla $(21 \%)$ y una pequeña cantidad en la cáscara (2.5\%). Por otra parte, el tratamiento de $200 \mathrm{mg} \mathrm{L}^{-1}$, presentó $67.43 \mathrm{mg} \mathrm{kg}^{-1}$, con valores de $78 \%$, $19 \%$ y $2.5 \%$, respectivamente. Mientras que en los frutos con fertilización comercial (GZ) la cantidad fue $57.6 \mathrm{mg} \mathrm{kg}^{-1}$, con valores de $77 \%$ (pulpa), $20 \%$ (semilla) y $2.5 \%$ (cascara) (Figura 2).

El tratamiento de $200 \mathrm{mg} \mathrm{L}^{-1} \mathrm{NPs} \mathrm{ZnO}$, fue el que presentó el mayor contenido de zinc en pulpa (52.96 $\mathrm{mg} \mathrm{kg}^{-1}$ ), superando al tratamiento control 24\%, y a GZ en 17\%. Sin embargo, a medida que se aplicó una mayor concentración los valores de zinc en pulpa disminuyeron en 6\%. En otros estudios se han reportado contenidos de zinc de $22 \mathrm{mg} \mathrm{kg}^{-1}$ peso seco en pulpa de melón (LópezZaplana et al., 2020). 


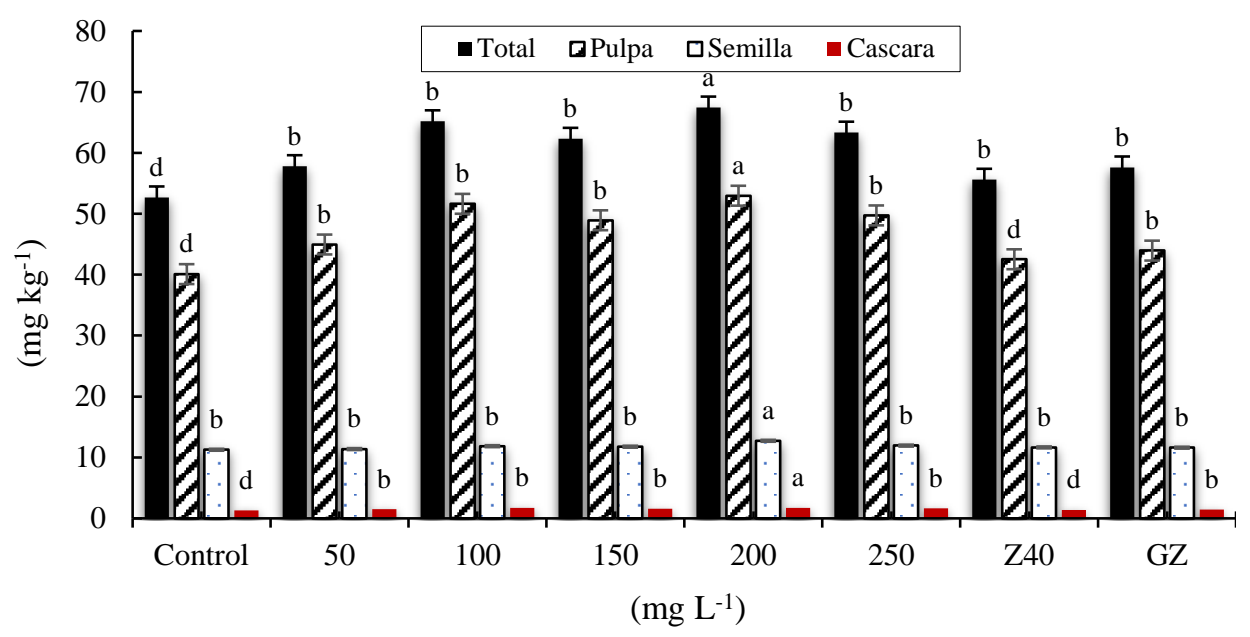

Figura 2. Contenido de zinc en cascara, pulpa y semillas de frutos de melón con aplicaciones foliares de nanopartículas de zinc y fertilizantes comerciales convencionales. Promedios con letras distintas indican diferencia significativa según la prueba de Tukey $(p \leq 0.05)$.

Por otra parte, Wang et al. (2019) encontraron que al aplicar NPs Cu se incrementó el contenido de cobre en granos de trigo entre $18.86 \%$ y $30.45 \%$ en comparación con el control y con una dosis de $500 \mathrm{mg} \mathrm{kg}^{-1}$ de NPs TiO. Rizwan et al. (2018) observaron que al aplicar Nanopartículas de Zn y $\mathrm{Fe}$ hubo un incremento en las concentraciones de ambos elementos en Triticum aestivum. El Instituto de Medicina (2002) reporta un requerimiento de $11 \mathrm{mg} \mathrm{día}^{-1}$ de zinc en adolescentes de 14 a 18 años y de $9.4 \mathrm{mg}$ para personas mayores de 19 años, no se recomienda ingerir dosis mayores de $25 \mathrm{mg}$, ya que puede causar anemia y deficiencia de cobre (Lenntech, 2021).

En este sentido, la utilización de NPs con zinc es una manera eficaz de enriquecer a los cultivos, ya que al trasladar $\mathrm{Zn}$ por tejidos vegetales se provocará una acumulación de este micronutrimento, lo que podría ayudar a solucionar la deficiencia de $\mathrm{Zn}$ en la dieta humana (Cakman y Kutman, 2018).

\section{Conclusiones}

La aplicación foliar de NPs $\mathrm{ZnO}$ mejora el rendimiento y la calidad comercial y nutracéutica de los frutos de melón. El rendimiento y la calidad del cultivo de melón depende de la concentración utilizada de NPs $\mathrm{ZnO}$ ya que dosis altas favorece el rendimiento y una mayor concentración de $\mathrm{Zn}$ en pulpa; en cambio on la aplicación de dosis intermedias de NPs $\mathrm{ZnO}$ se propicia una mayor acumulación de compuesto bioactivos. La aspersión foliar de NPs ZnO es una manera práctica de enriquecer los frutos de melón y mitigar las deficiencias de este micro nutrimento en la población.

\section{Agradecimientos}

Rubén Guadalupe Rivera-Gutiérrez agradece el apoyo económico brindado por el Consejo Nacional de Ciencia y Tecnología de México (CONACYT) para estudios de doctorado y a la asistencia técnica de los técnicos del Laboratorio de Análisis de Agua y Suelos del Instituto Tecnológico de Torreón (ITT), por su apoyo y patrocinio para realizar el proyecto y al CIQA por la donación de las nanopartículas. 


\section{Literatura citada}

Amarakoon, D. 2012. Guisantes de campo ricos en hierro, zinc y magnesio (Pisum sativum L.) con ácido fítico naturalmente bajo: una posible solución a base de alimentos para la desnutrición global de micronutrientes. Rev. Composic. Anális. Alimen. 27(1):8-13.

AOAC. 1995. Official Methods of Analysis. Association of official analytical chemists. Arlington VA, USA. 16 p.

Arellano, M.; Blundell, R. y Bonhomme, S. 2017. Dinámica de ingresos y consumo: un marco de datos de panel no lineal. Econometrica. 85(3):693-734. 10.3982/ecta13795.

Azam, M. M.; Eissa, A. H. A. and Hassan, A. H. 2015. Monitoring of change in cantaloupe fruit quality under pre-cooling and storage treatments. J. Food Process Technol. 6(12):1-6.

Bayer. 2018. Bayer cross design and seminis melón, una fresca opción para tu siembra de verano. Agronomic Spotligths. https://www.seminis.mx/melon-una-fresca-opcion-para-tu-siembra -de-verano/.

Beaulieu, J. C. 2007. Cambios de calidad en el melón durante el crecimiento, la maduración y en cubos recién cortados almacenados preparados a partir de frutas cosechadas en varios vencimientos J. Amer. Soc. Hort. Sci. 132(5):720-728.

Bower, J. H.; Latché, P. and Jean-Claude. 2002. Culture conditions and detachment of the fruit influence the effect of ethylene on the climateric respiration of melon. Postharvest Biol. Tec. 26(4):135-146.

Brand-Williams, W. C. and Berset, C. 1995. Use of free radical method to evaluuate antioxidant activity. Lebensm. Wiss. Technol. 28:25-30.

Cakmak, I. y K, U. B. 2017. Biofortificación agronómica de cereales con zinc: una revisión. Rev. Europea. Cienc. Suel. 69(1):172-180.

Cano, R. P.; Moreno, L. E.; Espinoza, J. J.; Jiménez, F. y Nava, U. 1992. Guía para cultivar melón en la comarca lagunera. Campo Experimental 'La Laguna' de INIFAP. Matamoros, Coahuila. 2-15 pp.

Chandra, P.; Sharma, R. K. and Arora, D. S. 2020. Antioxidant compounds from microbial sources: a review. Food Res. Int. 129 p.

Chang, J. y Lin, C. Y. 2001. Cinética de decoloración de una cepa recombinante de escherichia coli que alberga determinantes que decoloran azo-colorantes de rhodococcus sp. Biotechnol. Lett. 23:631-636. 10.1023/A: 1010306114286.

De-Rosa, M.; Monreal, C.; Schnitzer, M.; Walsh, R. y Sultan, Y. 2010. Nanotecnología en fertilizantes. Nat. Nanotechnol. 5(2):91-91.

Doner. L. W. 1981. Hiah-nerformance liouid chromatographic separation of ascorbic acid, erythorbic acid, dehydoascorbic acid, dehydroerythorbic acid, diketogulonic acid, and diketogluconic acid. Anal. Biochem. 115(1):225-230.

Fageria, N. K. 2016. The use of nutrients in crop plants. Boca raton: CRC press. 448 p. $10.1201 / 9781420075113$.

Faizan, M. H. and Shamsul. 2019. Effect of foliar spray of zno-nps on the physiological parameters and antioxidant systems of lycopersicon esculentum. Polish J. Nat. Sci. 34(6):87-105.

Faostat. 2020. Food and Agriculture Organization the United Nations Statistics. 2020 p. http://www.fao.org/faostat/en/\#home.

García, E. 2004. Modificaciones al sistema de clasificación climática de Köppen, adaptado a las condiciones de la República Mexicana. $5^{\text {a }}$ (Ed.). No. 6. Instituto de Geografía- Universidad Nacional Autónoma de México (UNAM). 97 p. 
García-Gómez, C.; Obrador, A.; González, D.; Babín, M. y Fernández, M. D. 2017. Efecto comparativo de $\mathrm{NP}$ de $\mathrm{ZnO}, \mathrm{ZnO}$ a granel y $\mathrm{ZnSO}_{4}$ en las defensas antioxidantes de dos especies de plantas que crecen en dos suelos agrícolas en condiciones de invernadero. Sci. Total Environ. 589(7):11-24.

García-López, J. N. M.; Olivares-Sáenz, E.; Lira-Saldivar, R. H.; Barriga-Castro, E. D.; VázquezAlvarado, R.; Rodríguez-Salinas, P. A. y Zavala-García, F. 2019. La aplicación foliar de nanopartículas de óxido de zinc y sulfato de zinc aumenta el contenido de compuestos bioactivos en pimientos habaneros. Plants. 8(254):1-20.

García-Mendoza, V.; Cano-Ríos, P. and Reyes-Carrillo, J. L. 2019. Harper-type melon hybrids have higher quality and longer postharvest life than commercial hybrids. Rev. Chapingo Ser. Hortic. 25(3):185-197. 10.5154/r.rchsh.2019.05.008.

Gawrońska, H. P.; Arkadiusz, S.; Pawlak, K.; Brama, K.; Miszczak, A.; Stankiewicz-Kosyl, M. and Gawronski, S. 2018. Platinum uptake, distribution and toxicity in Arabidopsis thaliana L. plants. Ecotox Environ Safe. 147:982-989. 10.1016/j.ecoenv.2017.09.065.

Ghosh, M.; Jana, A.; Sinha, S.; Jothiramajayam, M.; Nag, A.; Chakraborty, A.; Mukherjee, A. and Mukherjee, A. 2016. Effects of $\mathrm{ZnO}$ nanoparticles in plants: Cytotoxicity, genotoxicity, deregulation of antioxidant defenses, and cell-cycle arrest. Mutation research. Gen. Toxicol Environ. Mutagen. 807(1):25-32. 10.1016/j.mrgentox.2016.07.006.

Harborne, J. B. and Williams, C. A. 2000. Advances in flavonoid research since 1992. Phytochemistry. 55(6):481-504. 10.1016/s0031-9422(00)00235-1.

Ismail, H.; Chan, K. W.; Mariod, A. A. e Ismail, M. 2010. Contenido fenólico y actividad antioxidante de extractos metanólicos de melón (Cucumis melo). Química de los Alimentos. 119(2):643-647. 10.1016/ j.foodchem.2009.07.023.

Jeong, S. M.; Kim, S. Y.; Kim, D. R.; Jo, S. C.; Nam, K. C.; Ahn, D. U. y Lee, S. C. 2004. Efecto del tratamiento térmico sobre la actividad antioxidante de extractos de cáscaras de cítricos. Rev. Quím. Agríc. Aliment. 52(11):3389-3393. 10.1021 / jf049899k.

Jurkow, R.; Pokluda, R.; Sękara, A. y Kalisz, A. 2020. Impact of foliar application of some metal nanoparticles on antioxidant system in oakleaf lettuce seedlings. BMC Plant Biology. 20(290):1-12.

Khodakovskaya, M. V.; Silva, K.; Biris, A. S.; Dervishi, E. and Villagarcia, H. 2012. Carbon nanotubes induce growth enhancement of tobacco cells. ACS nano. 6(3):2128-2135. $10.1021 / \mathrm{nn} 204643 \mathrm{~g}$.

Kolenčík, M.; Ernst, D.; Komár, M.; Urík, M.; Šebesta, M.; Dobročka, E.; Černý, I.; Illa, R.; Kanike, R.; Qian, Y.; Feng, H.; Orlová, D. and Kratošová, G. 2019. Effect of foliar spray application of zinc oxide nanoparticles on quantitative, nutritional, and physiological parameters of foxtail millet (Setaria italica L.) under field conditions. Nanomaterials (Basel, Switzerland). 9(11):1559. 10.3390/nano9111559.

Lenntech. 2021. Ingesta diaria recomendada de vitaminas y minerales. https://www.lenntech.com/ recommended-daily-intake.htm.

López-Romaña, D.; Castillo, D.; Carlos y Diazgranados, D. 2010. El zinc en la salud humana. Revista Chilena de Nutrición. 37(2):240-247. 10.4067/S0717-75182010000200014.

López, V.; Santana, A. and Rodríguez, L. 2007. Sustainable development and corporate performance: A study based on the dow jones sustainability index. J. Bus. Ethics. 75(3):285-300. 10.1007/s10551-006-9253-8.

López-Vargas, E.; Ortega-Ortiz, H.; Cadenas-Pliego, G.; Alba-Romenus, K.; Cabrera-Fuente, M.; Benavides-Mendoza, A. y Juárez-Maldonado, A. 2018. La aplicación foliar de nanopartículas de cobre aumenta la calidad de la fruta y el contenido de compuestos bioactivos en tomates. Ciencias Aplicadas. 8(7):10-20. 10.3390 / app8071020. 
Maietti, A; Tedeschi, P.; Stagno, C.; Bordiga, M.; Travaglia, F.; Locatelli, M.; Arlorio, M. and Brandolini, V. 2012. Analytical traceability of melon (Cucumis melo var reticulatus): proximate composition, bioactive compounds, and antioxidant capacity in relation to cultivar, plant physiology state, and seasonal variability. J. Food Sci. 77(6):646-652. 10.1111/j.1750-3841.2012.02712.x.

Mallek-Ayadi, S.; Bahloul, N. y Kechaou, N. 2019. Caracterización, compuestos fenólicos y propiedades funcionales de los peelings de Cucumis melo L. Food Chem. 221(1):16911697. 10.1016 / j.foodchem.2016.10.117.

Mathpal, B.; Srivastava, P. C.; Shankhdhar, D. y Shankhdhar, S. C. 2015. Mejora de las actividades enzimáticas clave y la calidad del arroz bajo varios métodos de aplicación de zinc. Fisiología y Biología Molecular de las Plantas. 21(4):567-572. 10.1007/s 12298-015-0321-3.

Méndez-Argüello, B.; Vera-Reyes, I.; Mendoza-Mendoza, E.; García-Cerda, L. A.; Puente-Urbina, B. A. and Lira-Saldívar, R. H. 2016. Promoción del crecimiento en plantas de Capsicum annuum por nanopartículas de óxido de zinc. Nova Scientia. 8(17):140-156.

Morais, D.; Rotta, E. M.; Sargi, S. C.; Schmidt, E. M.; Bonafe, E. G.; Eberlin, M. N.; Sawaya, A. C. y Visentainer, J. V. 2015. Actividad antioxidante, fenólicos y UPLC ESI MS de extractos de diferentes partes de frutas tropicales y cáscaras procesadas. Food Res. Intern. 77(1):392-399.10.1016/j.foodres.2015.08.036.

Moreno-Reséndez, A.; García-Gutiérrez, L.; Cano-Ríos, P.; Martínez-Cueto, V.; MárquezHernández, C. y Rodríguez-Dimas, N. 2014. Desarrollo del cultivo de melón (Cucumis melo) con vermicompost bajo condiciones de invernadero. Ecosistemas y Recursos Agropecuarios. 1(2):163-173.

Mousavi, S. C. T.; Nguyen-Farmer, E. and Kellenberger, S. 2014. Measuring surface potential changes on leaves. Nature Protocols. 9(8):1997-2004. 10.1038/nprot.2014.136.

Nandhini, M. R.; Udayashankar, S. B.; Niranjana, S. R.; Søgaard, O.; Shetty, H. and Prakash, H. S, 2019. Biofabricated zinc oxide nanoparticles as an eco-friendly alternative for growth promotion and management of downy mildew of pearl millet. Crop Protection. 121(1):103112. 10.1016/j.cropro.2019.03.015.

Neeraja, C. N. B; Ram, S.; Hossain, F.; Hariprasanna, K.; Rajpurohit, B. S.; Prabhakar, L. T.; Prasad, K. S.; Sandhu, J. S. and Datta, S. K. 2017. Biofortification in cereals: progress and prospects. Current Sci. 113(6):1050-1057. 10.18520/cs/v113/i06/1050-1057.

Padayatty, S.; Katz, A.; Wang, Y.; Eck, P.; Kwon, O.; Lee, J.-H. y Levine, M. 2003. La vitamina C como antioxidante: evaluación de su papel en la prevención de enfermedades. Rev. Coleg. Americ. Nutric. 22(1):18-35. 10.1080/07315724.2003.10719272.

Prasad, R.; Shivay, Y. S. and Kumar, D. 2014. Chapter two agronomic biofortification of cereal grains with iron and zinc. In: Donald LS. (Ed) advances in agronomy. Academic Press. 55$91 \mathrm{pp}$.

Rajitha, B.; Rajarajeswari, V.; Sudhakar, P.; Naidu, N. V.; Prasad, T. y Reddy, B. 2020. Efecto de nutrientes de plantas a nanoescala en caracteres de plántulas de Blackgram (Vigna mungo (L.) Hepper). Rev. Farmacognosia y Fitoquímica. 9(2):670-672.

Ramírez-Barrón, S. N.; Sánchez-Valdés, S.; Puente-Urbina, B. A.; Martínez-Montemayor, S.; Esparza-González, S. C. Betancourt-Galindo, R. 2019. Preparación de un adhesivo sensible a la presión (PSA) con la incorporación de nanopartículas de ZnO. Estudio de sus propiedades fisicoquímicas y antimicrobianas. Rev. Mexic. Ingen. Biomédica. 40(1):201838. 10.17488/rmib.40.1.5. 
Reed, R.; Ladner, D. A.; Higgins, C. P.; Westerhoff, P. y Ranville, J. F. 2011. Solubilidad del óxido de nano-zinc en matrices importantes desde el punto de vista medioambiental y biológico. Toxicología y Química Ambiental. 31(1):93-99. 10.1002 etc.708.

Restrepo-Betancur y López-Marín, B. E. 2020. Biofortificación de tomate (Solanum lycopersicum L.) con cianocobalamina y micronutrientes aminoquelados (Zn, Fe). Rev. Esp, Nutr. Hum. Diet. 24(3):247-255. 10.14306/renhyd.24.3.1011.

Rizwan, M.; Ali, S.; Ali, B.; Adrees, M.; Arshad, M.; Hussain, A.; Zia, R. M. and Waris, A. A. 2018. Zinc and iron oxide nanoparticles improved the plant growth and reduced the oxidative stress and cadmium concentration in wheat. Chemosphere. 214(1):269-277. Doi:10.1016/j.chemosphere.2018.09.120.

Salgueiro, M. J.; Weill, R; Hernández-Triana, M.; Zubillaga, L. A.; Ruiz, C.; Vladimir, B. J. y Caro, R. 2004. Deficiencia de zinc en relación con el desarrollo intelectual y sexual. Rev. Cub. Sal. Públ. 30(2):1-13.

SIAP. 2021. Servicio de Información Agroalimentaria y Pesquera. Anuario Estadístico de la Producción Agrícola. https://nube.siap.gob.mx/cierreagricola/.

Singleton, V.; Orthofer, R. y Lamuela-Raventós, R. M. 1999. Análisis de fenoles totales y otros sustratos de oxidación y antioxidantes mediante reactivo folin-ciocalteu. Methods in Enzymology. 299:152-178. 10.1016/s0076-6879 (99) 99017-1.

Sturikova, H. K.; Huska, D.; Adam, V. 2018. Zinc, zinc nanoparticles and plants. J. Hazardous Materials. 349:101-110. 1016/j.jhazmat.2018.01.040.

Subbaiah, L. P.; Tollamadugu, N. V.; Gilidiará, K.; Timmavajjula, S. P.; Reddy, B. and Pradeep, T. N. 2016. Effects of nanoparticulate delivery of zinc on growth, productivity, and zinc biofortification in maize (Zea mays L.). J. Agr. Food Chem. 64(19):3778-3788. Doi:10.1021/acs.jafc.6b00838.

Tadmor, Y.; Burger, J.; Yaakov, I.; Feder, A.; Libhaber, S. E.; Portnoy, V.; Meir, A.; Tzuri, G.; Sa'ar, U.; Rogachev, I.; Aharoni, A.; Abeliovich, H.; Schaffer, A. A.; Lewinsohn, E. and Katzir, N. 2010. Genetics of flavonoid, carotenoid, and chlorophyll pigments in melon fruit rinds. J. Agr. Food Chem. 58(19):10722-10728. 10.1021/jf1021797.

Trinidad, S. 1999. Fertilización foliar, un respaldo importante en el rendimiento de los cultivos. Terra Latinoam. 17(3):247-255.

Wang, M.; Liu, X.; Hu, J.; Li, J. y Huang, J. 2015. El óxido nano-férrico promueve el crecimiento de la sandía. Rev. Biomateriales y Nanobiotecnología. 6(3):160-167. 10.4236/jbnb. 2015.63016.

Wang, Y.; Jiang, F.; Ma, C.; Rui, Y.; Tsang, D. C. W. y Xing, B. 2019. Efecto de nanopartículas de óxido metálico sobre aminoácidos en granos de trigo (Triticum aestivum) en un estudio de ciclo de vida. J. Environ. Manage. 241:319-327. 10.1016 / j.jenvman.2019.04.041.

Yin, X.; Yuan, L.; Liu, Y. y Lin, Z. 2012. Phytoremediation and Biofortification: Two Sides of One Coin. Berlin, NY: Springer. 1-31 p. doi:10.1007/978-94-007-1439-7_1.

Zhishen, J. M. y Jianming, W. 1999. La determinación del contenido de flavonoides en la morera y sus efectos depuradores sobre los radicales superóxidos. Food Chem. 64(1):555-559. 